\title{
SIMILARITY SOLUTIONS FOR BOUNDARY LAYER EQUATIONS OF A POWEL-EYRING FLUID
}

\author{
Tasawar Hayat ${ }^{1,2}$, Mehmet Pakdemirli ${ }^{3}$ and Yiğit Aksoy ${ }^{3}$ \\ ${ }^{1}$ Department of Mathematics, Quaid-i Azam University, Islamabad, Pakistan \\ ${ }^{2}$ Department of Mathematics, King Saud University, P.O. Box 2455, Riyadh 11451, \\ Saudi Arabia \\ ${ }^{3}$ Department of Mechanical Engineering, Celal Bayar University, 45140, Muradiye, \\ Manisa, Turkey \\ mpak@cbu.edu.tr
}

\begin{abstract}
Boundary layer equations are derived for the first time for the Powel-Eyring fluid model, a non-Newtonian model proposed for pseudoplastic behavior. Using a scaling symmetry of the equations, partial differential system is transferred to an ordinary differential system. Resulting equations are numerically solved using a finite difference algorithm. Effects of non-Newtonian parameters on the solutions are discussed.
\end{abstract}

Key Words- Non-Newtonian Fluid, Powel-Eyring Fluid, Boundary Layer Theory, Similarity Transformations

\section{INTRODUCTION}

Many fluids like biological fluids, shampoo, yoghurt, tomato sauce, paints, lubricants, polymeric fluids do not obey the linear stress-velocity gradient relationship in Newtonian fluid theory. Several non-Newtonian fluid models were therefore proposed to explain the complex behavior. Usually, the stress constitutive relations of such models inherit complexities which lead to highly nonlinear equations of motion with many terms. To simplify the extremely complex equations, one alternative is to use boundary layer theory which is known to effectively reduce the complexity of NavierStokes equations and reduce drastically the computational time. Since there are many non-Newtonian models and new models are being proposed continuously, boundary layer theory for each proposed model also appear in the literature. It is beyond the scope of this work to review vast literature on the boundary layers of non-Newtonian fluids. A limited work on the topic can be referred as examples [1-23].

In this work, boundary layer equations are developed for the first time for the Powel-Eyring fluid. One of the interesting features of Powell-Eyring fluids is that, the stress constitutive relation of such fluids can be derived using the kinetic theory of liquids. This model correctly reduces to Newtonian behavior for low and high shear rates. Scaling symmetry is well known to exist for boundary layer type problems leading to useful solutions and for this reason, the specific form of the scaling symmetry which leaves the equations invariant is determined. Using the symmetry, the partial differential system is transformed into an ordinary differential system. See [13-15, 23- 
25] for applications of scaling symmetries to various problems. Resulting ordinary differential system is numerically solved by a finite difference algorithm. Effect of nonNewtonian parameters on the velocity profiles are shown in the graphs.

Some of the recent work on Powel-Eyring fluids is as follows: Yürüsoy [26] studied a slider bearing lubricated with a Powel-Eyring fluid. Islam et al. [27] reconsidered the same problem and found solutions with homotopy perturbation method which verified the results of [26] obtained by perturbation method. Ai and Vafai [28] investigated Stokes second problem for eight different non-Newtonian fluid models including Powel-Eyring model. The boundary layer equations given in this work is new and do not exist in the previous literature.

\section{BOUNDARY LAYER EQUATIONS}

The Cauchy stress tensor for Powel-Eyring fluid is $\mathbf{T}=-p \mathbf{I}+\mathbf{S}$

where

$\mathbf{S}=\mu(\nabla \mathbf{V})+\frac{1}{\beta} \sinh ^{-1}\left(\frac{1}{c} \nabla \mathbf{V}\right)$

where $\mathbf{V}$ is the velocity vector. Steady-state, two dimensional, incompressible equations of motion including mass conservation can be written as

$$
\begin{gathered}
\frac{\partial u^{*}}{\partial x^{*}}+\frac{\partial v^{*}}{\partial y^{*}}=0 \\
\rho\left(u^{*} \frac{\partial u^{*}}{\partial x^{*}}+v^{*} \frac{\partial u^{*}}{\partial y^{*}}\right)=-\frac{\partial p^{*}}{\partial x^{*}}+\frac{\partial S_{x x}}{\partial x^{*}}+\frac{\partial S_{x y}}{\partial y^{*}} \\
\rho\left(u^{*} \frac{\partial v^{*}}{\partial x^{*}}+v^{*} \frac{\partial v^{*}}{\partial y^{*}}\right)=-\frac{\partial p^{*}}{\partial y^{*}}+\frac{\partial S_{y x}}{\partial x^{*}}+\frac{\partial S_{y y}}{\partial y^{*}}
\end{gathered}
$$

where $\mathrm{x}^{*}$ is the spatial coordinate along the surface, $\mathrm{y}^{*}$ is vertical to it, $\mathrm{u}^{*}$ and $\mathrm{v}^{*}$ are the velocity components in the $\mathrm{x}^{*}$ and $\mathrm{y}^{*}$ coordinates.

To calculate the stress components in (2), the function is approximated first

$\sinh ^{-1}\left(\frac{1}{c} \nabla \mathbf{V}\right) \cong \frac{1}{c}(\nabla \mathbf{V})-\frac{1}{6}\left(\frac{1}{c} \nabla \mathbf{V}\right)^{3}$

and then substituted into (2)

$$
\mathbf{S} \cong\left(\mu+\frac{1}{\beta c}\right)(\nabla \mathbf{V})-\frac{1}{6 \beta c^{3}}(\nabla \mathbf{V})^{3}
$$

The shear stress components are inserted into the equations of motion and the usual boundary layer assumptions are made i.e. $x{ }^{*} \sim O(1), y^{*} \sim O(\delta), u^{*} \sim O(1), v^{*} \sim O(\delta)$. The highest order terms are retained and the momentum equations become

$$
\begin{aligned}
& \rho\left(u^{*} \frac{\partial u^{*}}{\partial x^{*}}+v^{*} \frac{\partial u^{*}}{\partial y^{*}}\right)=-\frac{\partial p^{*}}{\partial x^{*}}+\left(\mu+\frac{1}{\beta c}\right) \frac{\partial^{2} u^{*}}{\partial y^{* 2}}-\frac{1}{2 \beta c^{3}}\left(\frac{\partial u^{*}}{\partial y^{*}}\right)^{2} \frac{\partial^{2} u^{*}}{\partial y^{* 2}} \\
& 0=\frac{\partial p^{*}}{\partial y^{*}}
\end{aligned}
$$


from which dependence of pressure on $\mathrm{y}^{*}$ is eliminated. In deriving the equations $\mu \sim \mathrm{O}\left(\delta^{2}\right), 1 / \beta \sim \mathrm{O}(\delta)$ and $1 / \mathrm{c} \sim \mathrm{O}(\delta)$ assumptions are made. Dimensionless variables and parameters are defined as follows

$$
\begin{aligned}
& x=\frac{x^{*}}{L}, \quad y=\frac{y^{*}}{L}, \quad u=\frac{u^{*}}{V}, \quad v=\frac{v^{*}}{V}, \quad p=\frac{p^{*}}{\rho V^{2}} \\
& \varepsilon_{1}=\frac{\mu}{\rho V L}, \quad \varepsilon_{2}=\frac{1}{\rho \beta V^{2}}, \quad \varepsilon_{3}=\frac{V}{c L}
\end{aligned}
$$

where $L$ is a characteristic length and $V$ is a reference velocity. Expressing the pressure in terms of outer velocity, the final dimensionless boundary layer equations become

$$
\begin{gathered}
\frac{\partial u}{\partial x}+\frac{\partial v}{\partial y}=0 \\
u \frac{\partial u}{\partial x}+v \frac{\partial u}{\partial y}=-\frac{d p}{d x}+\left(\varepsilon_{1}+\varepsilon_{2} \varepsilon_{3}\right) \frac{\partial^{2} u}{\partial y^{2}}-\frac{1}{2} \varepsilon_{2} \varepsilon_{3}^{3}\left(\frac{\partial u}{\partial y}\right)^{2} \frac{\partial^{2} u}{\partial y^{2}}
\end{gathered}
$$

The classical boundary conditions for the problem are

$$
u(x, 0)=0, \quad v(x, 0)=0, \quad u(x, \infty)=U(x)
$$

For $\varepsilon_{2}=0$ or $\varepsilon_{3}=0$, the equations reduce to those of Newtonian fluid.

\section{SIMILARITY TRANSFORMATIONS}

Scaling symmetry is one of the most common symmetries producing useful solutions in boundary layer type equations [13-15, 23]. All variables are rescaled as follows

$\bar{x}=\lambda^{a} x, \quad \bar{y}=\lambda^{b} y, \quad \bar{u}=\lambda^{c} u, \quad \bar{v}=\lambda^{d} v, \quad \bar{U}=\lambda^{e} U$

Substituting (14) into (11) and (12) and requiring that the equations be invariant under the transformation yields

$b+c-a-d=0, \quad 2 c-2 e=0, \quad 2 b+c-a=0, \quad 4 b-c-a=0$

All parameters are solved in terms of parameter $b$

$a=3 b, \quad c=b, \quad d=-b, \quad e=b$

The associated equations for this transformation which define similarity variables are

$$
\frac{d x}{3 x}=\frac{d y}{y}=\frac{d u}{u}=\frac{d v}{-v}=\frac{d U}{U}
$$

The similarity variable and functions are

$$
\xi=\frac{y}{x^{1 / 3}}, \quad u=x^{1 / 3} f(\xi), \quad v=\frac{g(\xi)}{x^{1 / 3}}, \quad U=x^{1 / 3}
$$

Substituting all into the boundary layer equations yields the ordinary differential system

$f-\xi f^{\prime}+3 g^{\prime}=0$

$f^{2}-\xi f f^{\prime}+3 g f^{\prime}=1+3\left(\varepsilon_{1}+\varepsilon_{2} \varepsilon_{3}\right) f^{\prime \prime}-\frac{3}{2} \varepsilon_{2} \varepsilon_{3}^{3}\left(f^{\prime}\right)^{2} f^{\prime \prime}$

The boundary conditions also transform

$$
f(0)=0, \quad g(0)=0, \quad f(\infty)=1
$$




\section{NUMERICAL RESULTS}

Equations (19) and (20) are numerically integrated using a finite difference scheme subject to the boundary conditions (21). In Figure 1, f function and in Figure 2, $\mathrm{g}$ function related to the $\mathrm{x}$ and $\mathrm{y}$ components of the velocities are drawn for different $\varepsilon_{1}$ parameters. Boundary layer becomes thicker for an increase in $\varepsilon_{1}$. There is an increase in y component of velocity which is negative over the whole domain for an increase in $\varepsilon_{1}$ as can be seen from Figure 2. A similar trend is observed for parameter $\varepsilon_{2}$ (See Figures 3 and 4). The last parameter $\varepsilon_{3}$ has also an effect of thickening of the boundary layers as can be seen from Figure 5 in the parameter range considered. The increase in y component of velocity as $\varepsilon_{3}$ increases is observed from Figure 6 . Note that for higher values of $\varepsilon_{3}$, it becomes extremely hard to numerically integrate the equations. A bigger $\varepsilon_{3}$ may also violate the validity of the Taylor series approximation for the stress tensor.

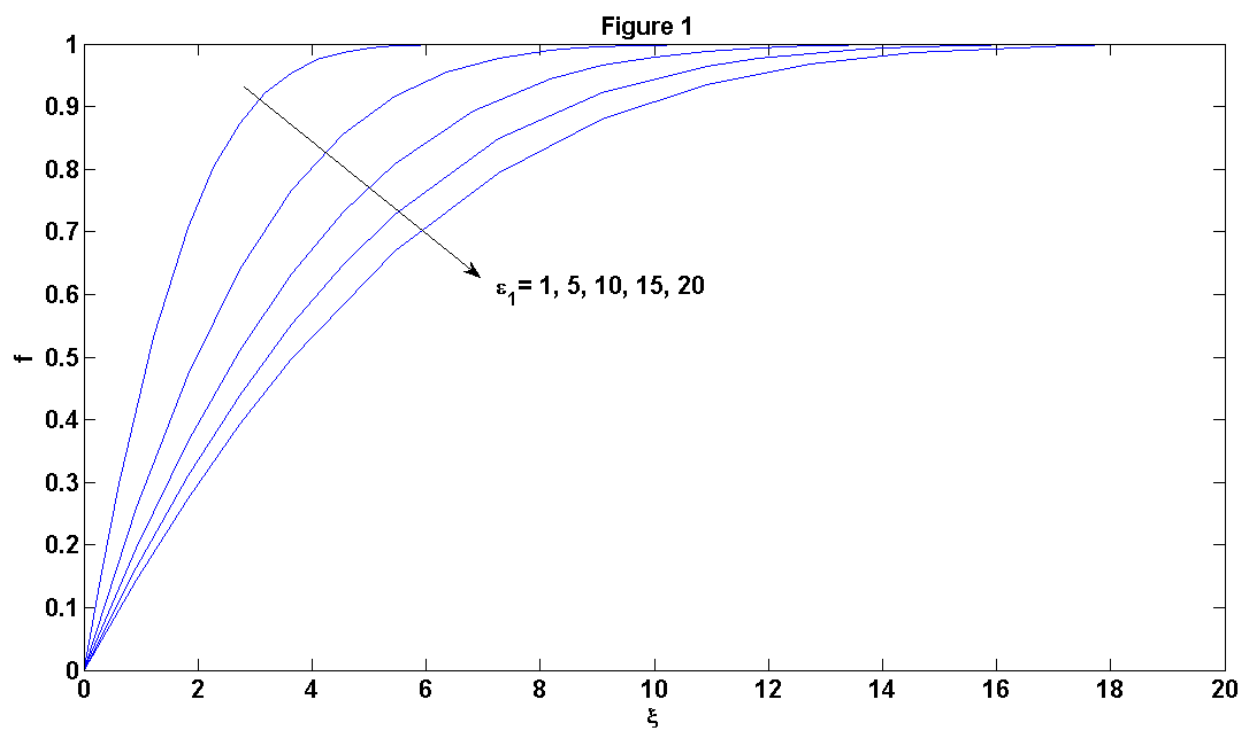

Figure 1. Effect of $\varepsilon_{1}$ parameter on the similarity function $f$ related to the x component of velocity $\left(\varepsilon_{2}=1, \varepsilon_{3}=1\right)$. 


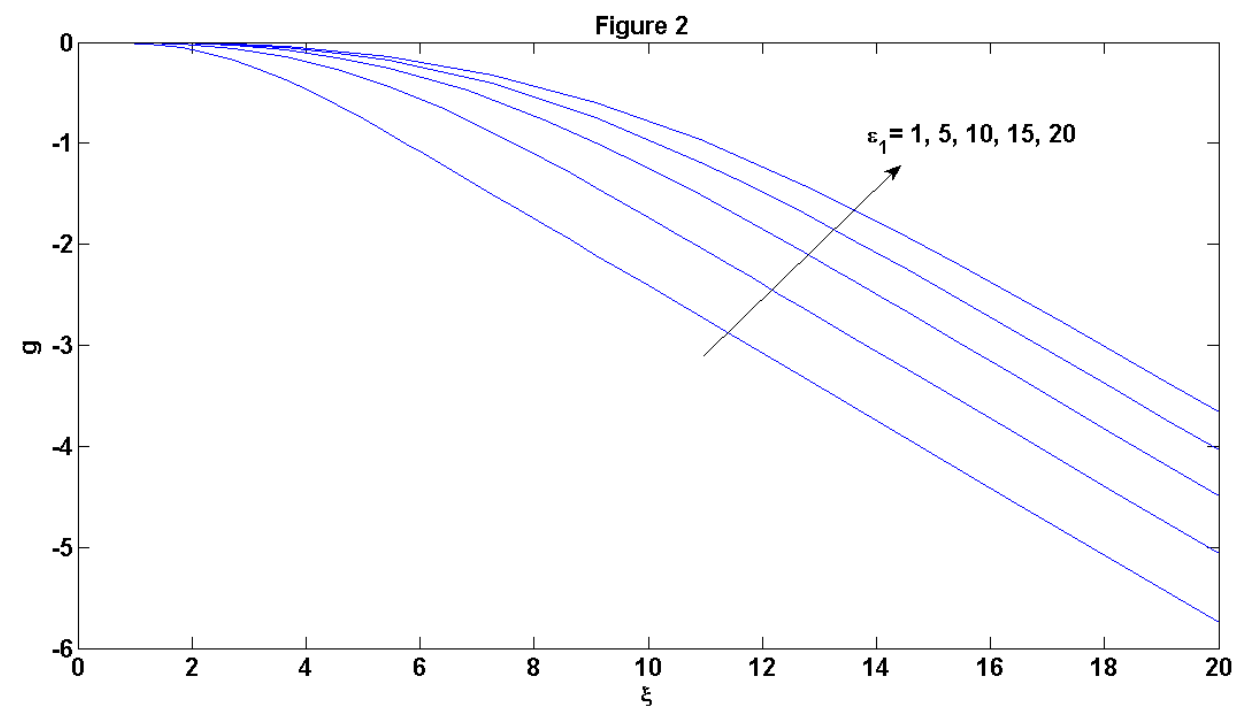

Figure 2. Effect of $\varepsilon_{1}$ parameter on the similarity function $g$ related to the y component of velocity $\left(\varepsilon_{2}=1, \varepsilon_{3}=1\right)$.

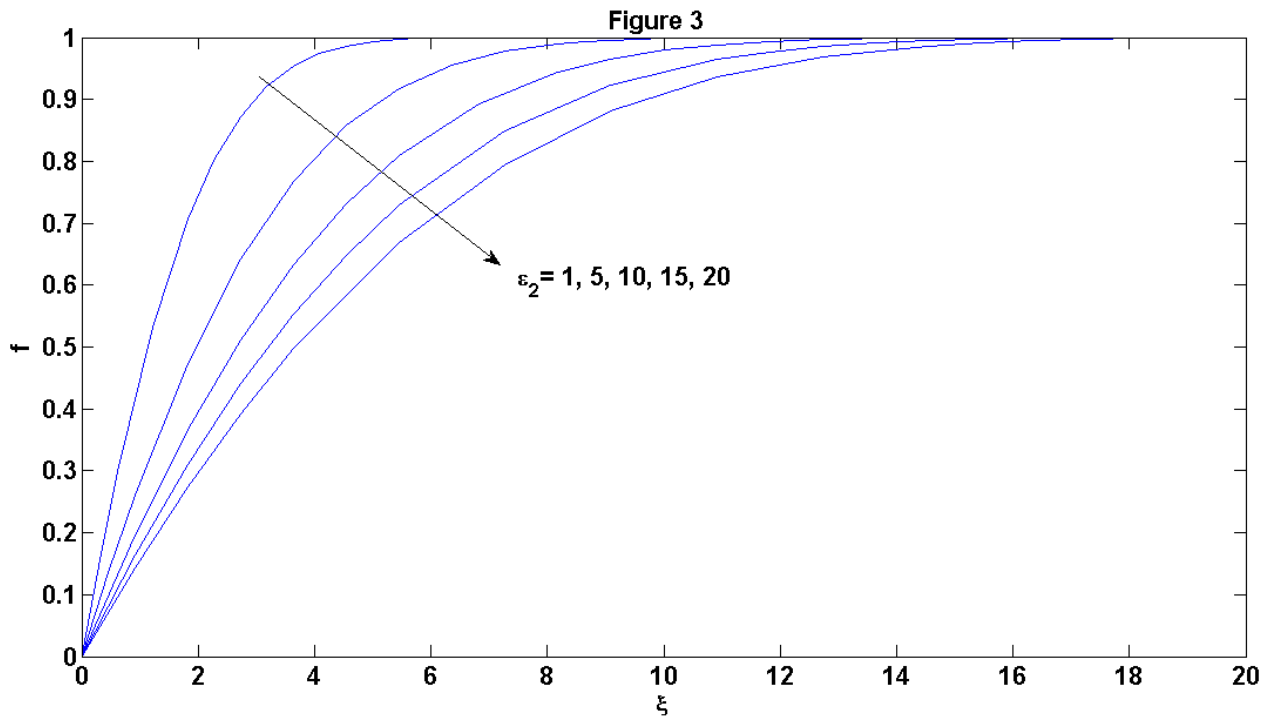

Figure 3. Effect of $\varepsilon_{2}$ parameter on the similarity function $\mathrm{f}$ related to the $\mathrm{x}$ component of velocity $\left(\varepsilon_{1}=1, \varepsilon_{3}=1\right)$. 


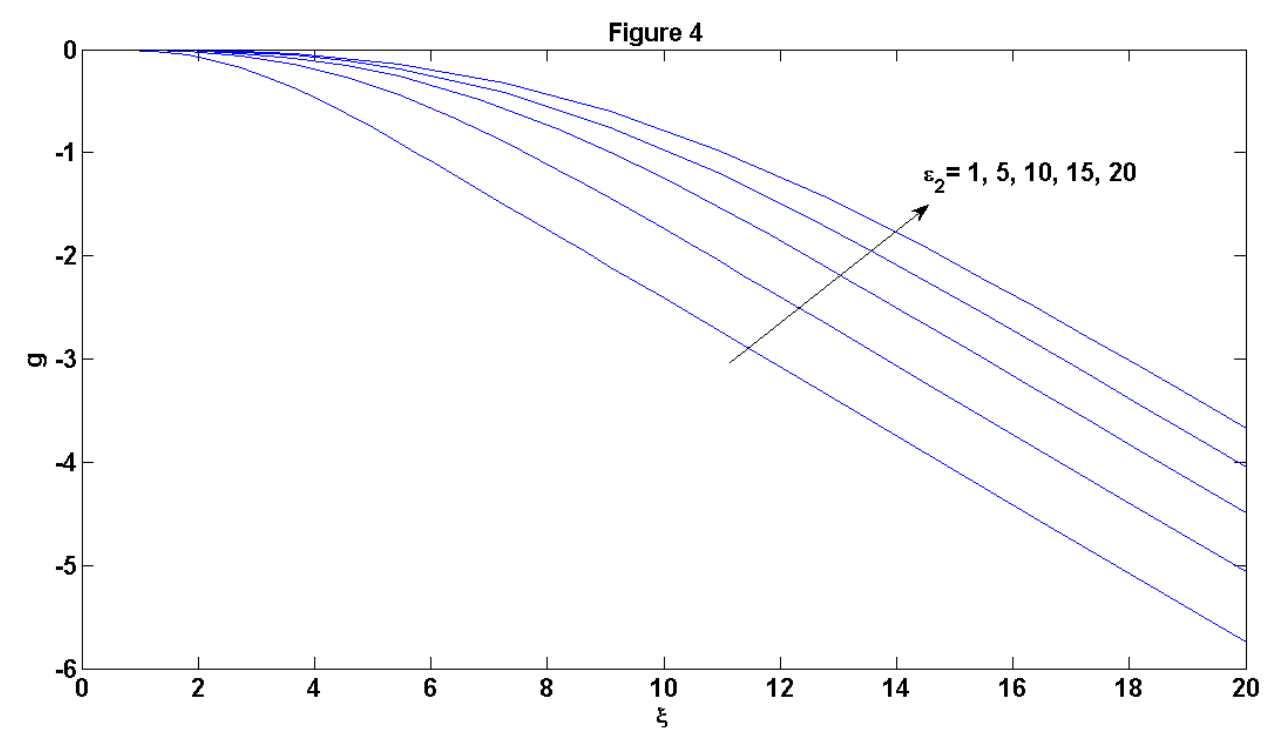

Figure 4. Effect of $\varepsilon_{2}$ parameter on the similarity function $g$ related to the y component of velocity $\left(\varepsilon_{1}=1, \varepsilon_{3}=1\right)$.

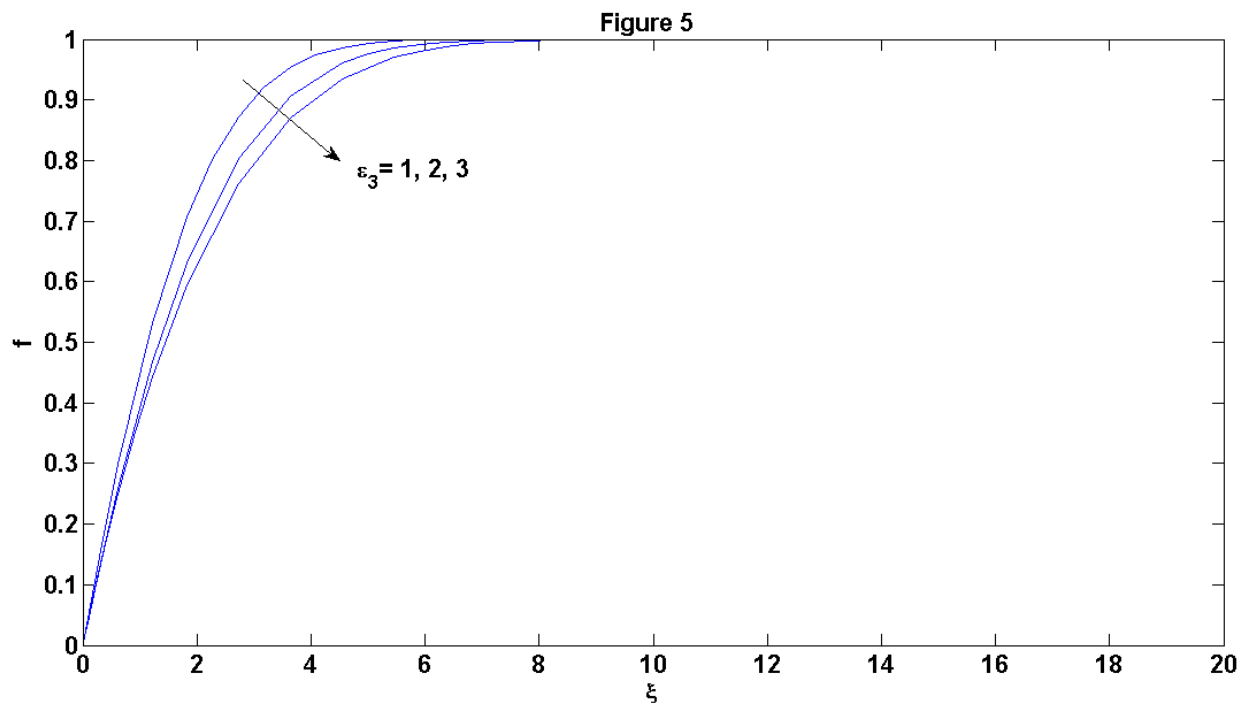

Figure 5. Effect of $\varepsilon_{3}$ parameter on the similarity function $\mathrm{f}$ related to the $\mathrm{x}$ component of velocity $\left(\varepsilon_{1}=1, \varepsilon_{2}=1\right)$. 


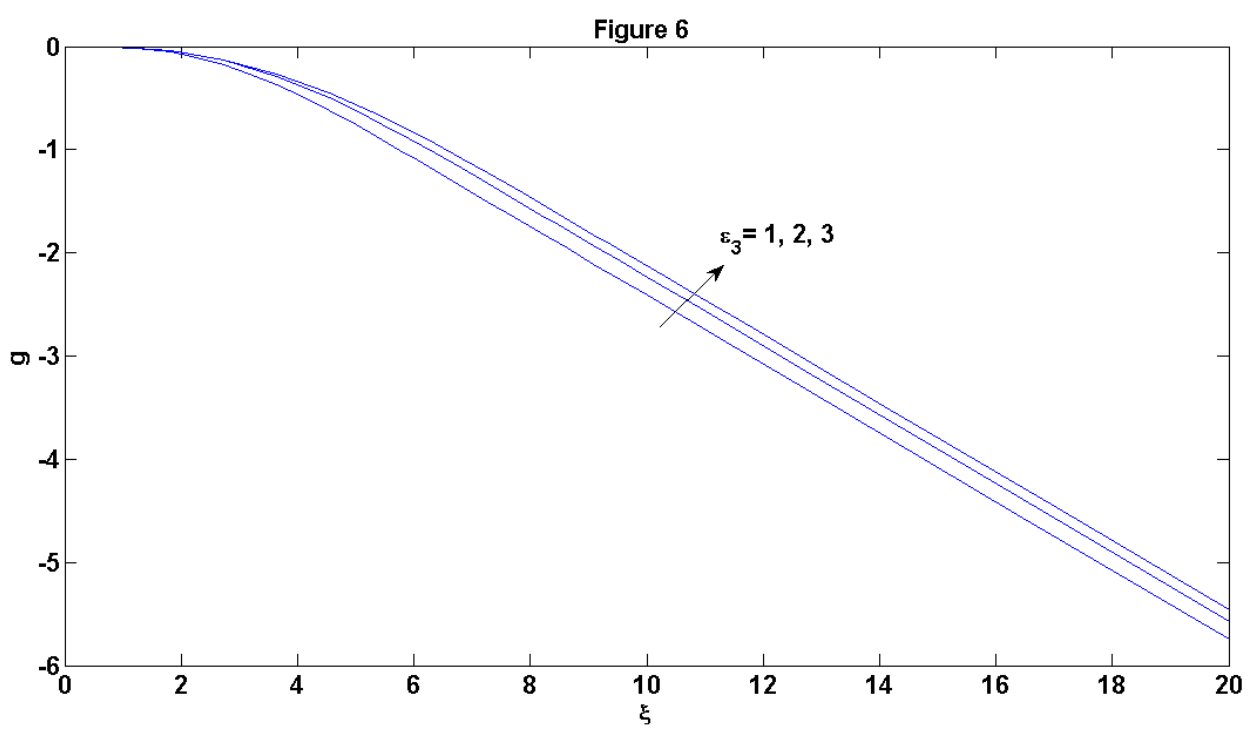

Figure 6. Effect of $\varepsilon_{3}$ parameter on the similarity function g related to the y component of velocity $\left(\varepsilon_{1}=1, \varepsilon_{2}=1\right)$.

Note the definitions of dimensionless parameters given in equation (10). An increase in the viscosity $\mu$ would yield an increase in parameter $\varepsilon_{1}$. It is therefore natural for the boundary layer to become wider for a higher viscosity value. More energy will be dissipated near the boundary which in turn causes the outer velocity to resume higher from the boundary. The rheological parameter $\beta$ has an inverse relation with dimensionless parameter $\varepsilon_{2}$. An increase in $\varepsilon_{2}$ means a decrease in $\beta$. The same applies to the relationship between $\varepsilon_{3}$ and the rheological parameter $\mathrm{c}$ also.

\section{CONCLUDING REMARKS}

Boundary layer equations of Powel-Eyring fluids are derived for the first time. Using a scaling transformation, the partial differential system is converted into an ordinary differential system which is solved numerically using a finite difference scheme. Effects of rheological parameters on the boundary layers are discussed in detail. It is found that an increase in all the dimensionless parameters $\varepsilon_{1}, \varepsilon_{2}$ and $\varepsilon_{3}$ causes the boundary layers to thicken.

Acknowledgements- This work is completed during mutual short visits of Tasawar Hayat to Turkey and Mehmet Pakdemirli to Pakistan. Funding supports of TUBITAK of Turkey and HEC of Pakistan are highly appreciated. 


\section{REFERENCES}

1. A. Acrivos, M. J. Shahand and E. E. Petersen, Momentum and heat transfer in laminar boundary layer flows of non-Newtonian fluids past external surfaces, AIChE Journal 6, 312-317, 1960.

2. W. R. Schowalter, The application of boundary-layer theory to power-law pseudoplastic fluids: Similarity solutions, AIChE Journal 6, 25-28, 1960.

3. G. D. Bizzell, J. C. Slattery, Non-Newtonian boundary layer flow, Chemical Engineering Science 17, 777-781, 1962.

4. A. C. Srivastava, The flow of a non-Newtonian fluid near a stagnation point, ZAMP 9, 80-84, 1958.

5. D. W. Beard and K. Walters, Elastico-viscous boundary layer flows, Proceedings of Cambridge Philosophical Society 60, 667-674, 1964.

6. J. Astin, R. S. Jones and P. Lockyer, Boundary layers in non-Newtonian fluids, Journal Mechanique 12, 527-539, 1973.

7. K. R. Rajagopal A. S. Gupta and A. S. Wineman, On a boundary layer theory for non-Newtonian fluids, International Journal of Engineering Science 18, 875-883, 1980.

8. K. R. Rajagopal A. S. Gupta and T. Y. Na, A note on the Falkner Skan flow of a nonNewtonian fluid, International Journal of Non-Linear Mechanics 18, 313-319, 1983.

9. V. K. Garg and K. R. Rajagopal, Flow of a non-Newtonian fluid past a wedge, Acta Mechanica 88, 113-123, 1991.

10. M. Massoudi and M. Ramezan, Effect of injection or suction on the Falkner Skan flows of second grade fluids, International Journal of Non-Linear Mechanics 24, 221227, 1989.

11. V. K. Garg and K. R. Rajagopal, Stagnation point flow of a non-Newtonian fluid, Mechanics Research Communications 17, 415-421, 1990.

12. M. Pakdemirli and E. S. Şuhubi, Boundary layer theory for second order fluids, International Journal of Engineering Science 30, 523-532, 1992.

13. M. Pakdemirli, The boundary layer equations of third grade fluids, International Journal of Non-Linear Mechanics 27, 785-793, 1992.

14. M. Pakdemirli, Boundary layer flow of power-law fluids past arbitrary profiles, IMA Journal of Applied Mathematics 50, 133-148, 1993.

15. M. Pakdemirli, Conventional and multiple deck boundary layer approach to second and third grade fluids, International Journal of Engineering Science 32, 141-154, 1994.

16. R. K. Bhatnagar, G. Gupta and K. R. Rajagopal, Flow of an Oldroy-B fluid due to a stretching sheet in the presence of a free stream velocity, International Journal of NonLinear Mechanics 30, 391-405, 1995.

17. T. Hagen and M. Renardy, Boundary layer analysis of the Phan-Thien-Tanner and Giesekus model in high Weissenberg number flow, Journal of Non-Newtonian Fluid Mechanics 73, 181-189, 1997.

18. M. Renardy, High Weissenberg number boundary layers for the upper convected Maxwell fluid, Journal of Non-Newtonain Fluid Mechanics 68, 125-132, 1997.

19. M. Yürüsoy and M. Pakdemirli, Symmetry reductions of unsteady threedimensional boundary layers of some non-Newtonian fluids, International Journal of Engineering Science 35, 731-740, 1997. 
20. M. Yürüsoy and M. Pakdemirli, Group classissification of a non-Newtonian fluid model using classical approach and equivalence transformations, International Journal of Non-Linear Mechanics 34, 341-346, 1999.

21. Y. Aksoy, M. Pakdemirli and C. M. Khalique, Boundary layer equations and stretching sheet solutions for the modified second grade fluid, International Journal of Engineering Science 45, 829-841, 2007.

22. M. Yürüsoy, Group classification of three-dimensional boundary layer equations of a class of non-Newtonian fluids, Applied Mathematics and Computation 157, 849-859, 2004.

23. M. Pakdemirli, Similarity analysis of boundary layer equations of a class of nonNewtonian fluids, International Journal of Non-Linear Mechanics 29, 187-196, 1994.

24. M. Pakdemirli and M. Yürüsoy, Similarity transformations for partial differential equations, SIAM Review 40, 96-101, 1998.

25. M. Pakdemirli and A. Z. Şahin, Similarity Analysis of a Nonlinear Fin Equation, Applied Mathematics Letters 19, 378-384, 2006.

26. M. Yürüsoy, A Study of Pressure Distribution of a Slider Bearing Lubricated with Powell-Eyring Fluid, Turkish Journal of Engineering and Environmental Sciences 27, 299-304, 2003.

27. S. Islam, A. Shah, C. Y. Zhou and I. Ali, Homotopy perturbation analysis of slider bearing with Powell-Eyring fluid, ZAMP 60, 1178-1193, 2009.

28. L. Ai and K. Vafai, An Investigation of Stokes' Second Problem for NonNewtonian Fluids, Numerical Heat Transfer, Part A 47, 955-980, 2005. 